\title{
Association of Neutrophil-Lymphocyte Ratio and the Presence of Neonatal Sepsis
}

\author{
Tiewei Li $\mathbb{D},{ }^{1}$ Geng Dong, ${ }^{1}$ Min Zhang, ${ }^{1}$ Zhe Xu, ${ }^{1}$ Yidi Hu, ${ }^{1}$ Bo Xie, ${ }^{1}$ Yuewu Wang $\mathbb{D},{ }^{2}$ \\ and Bangli $\mathrm{Xu} \mathbb{i}^{3}$ \\ ${ }^{1}$ Zhengzhou Key Laboratory of Children's Infection and Immunity, Children's Hospital Affiliated to Zhengzhou University, \\ Henan Children's Hospital, Zhengzhou Children's Hospital, Zhengzhou, China \\ ${ }^{2}$ The Engineering Research Center for New Drug Screening, Inner Mongolia Medical University, Hohhot, China \\ ${ }^{3}$ Department of Neonatology, Children's Hospital Affiliated to Zhengzhou University, Henan Children's Hospital, \\ Zhengzhou Children's Hospital, Zhengzhou, China
}

Correspondence should be addressed to Tiewei Li; litieweind@163.com, Yuewu Wang; 20090029@immu.edu.cn, and Bangli Xu; banglixu@sina.com

Received 19 July 2020; Revised 6 November 2020; Accepted 17 November 2020; Published 3 December 2020

Academic Editor: Cinzia Milito

Copyright ( $\odot 2020$ Tiewei Li et al. This is an open access article distributed under the Creative Commons Attribution License, which permits unrestricted use, distribution, and reproduction in any medium, provided the original work is properly cited.

\begin{abstract}
The neutrophil-lymphocyte ratio (NLR) is an emerging risk factor of sepsis that is receiving increasing attention. However, the relationship between NLR and the presence of sepsis in neonates is poorly studied. Here, we retrospectively recruited 1480 neonates and collected and analyzed relevant clinical and laboratory data. According to the International Pediatric Sepsis Consensus, 737 neonates were diagnosed with sepsis, and 555 neonates were suspected for having infection. Neonates with hyperbilirubinemia $(n=188)$ served as controls. Neonates with sepsis had significantly elevated neutrophil counts and NLR $(P<0.001)$. The proportion of neonates with sepsis increased significantly from $41.6 \%$ when NLR $<0.91$ to $66.2 \%$ when NLR $>$ 1.88 group $(P<0.001)$. Multiple logistic regression analysis showed that NLR was an independent risk factor for the presence of neonatal sepsis. Receiver operating characteristic (ROC) curve analysis showed that the optimal cut-off value NLR for predicting the presence of neonatal sepsis was 1.62 (area under curve $(\mathrm{AUC})=0.63,95 \% \mathrm{CI} 0.60-0.66, P<0.001$ ). In conclusion, our data suggest that elevated NLR levels are associated with a higher neonatal sepsis risk.
\end{abstract}

\section{Introduction}

Neonatal sepsis is one of the leading causes of morbidity and mortality in newborn infants [1] and is an important global public health issue [2]. Fleischmann-Struzek et al. reported that approximately 2202/100,000 livebirths develop neonatal sepsis, with mortality between $11 \%$ and $19 \%$ [3]. Early diagnosis and treatment of neonatal sepsis can help to prevent severe and life-threatening complications and subsequently reduce mortality. Clinical signs of neonatal sepsis include feeding intolerance, tachycardia, respiratory distress, pneumonia, and temperature instability, but these clinical symptoms often overlap with other noninfectious conditions making the diagnosis of neonatal sepsis difficult [1]. The gold standard for diagnosis of neonatal sepsis is blood culture [4], but blood cultures generally has a long waiting period, and the rate of positive cultures is low due to the small volume of blood used for inoculation and to contamination or antibiotics used before blood culture $[5,6]$. It is clear that better predictors are needed to diagnose neonatal sepsis. Circulating blood biomarkers that may be useful in the early diagnosis of neonatal sepsis have been processed [7].

Sepsis is a systemic inflammatory response syndrome caused by infection, and inflammation plays an important role in the initiation and progression of sepsis. White blood cells and their subpopulations are vital in immune system defenses against pathogen infection. Many clinical studies have shown that neutrophil counts, lymphocyte counts, and the neutrophil to lymphocyte ratio (NLR) are predictors of sepsis [8-10]. NLR is considered to be more stable than 
absolute neutrophil or lymphocyte counts as both neutrophil and lymphocyte counts are included in the calculation [11]. NLR has attracted substantial attention due to its potential as a new risk factor for sepsis [12-14]. However, most studies on the relationship between NLR and sepsis are conducted in adult patients [8], and there have only been a few reports on the association between NLR and neonatal sepsis, all with a relatively small sample sizes [15-18]. Therefore, the aim of this study is to evaluate the relationship between NLR and neonatal sepsis in a relatively large neonatal population.

\section{Materials and Methods}

2.1. Study Design and Population. A hospital-based retrospective case-control study was conducted from January 2016 to December 2019 in Henan Children's Hospital. A total 1480 neonates were included in the study, and clinical and laboratory data were collected. Patients with the following conditions were excluded: (1) missing total and differential leukocyte counts and (2) other diseases, such as hematological system diseases, major congenital malformation, and cyanotic congenital heart disease. The study protocol complied with the Declaration of Helsinki and was approved by the hospital ethics review board. Written informed consent was obtained from all the participants.

2.2. Clinical Evaluation and Definition. The diagnosis of clinical neonatal infection and sepsis was made by two independent doctors according to the International Pediatric Sepsis Consensus [4]. Infection was defined as a suspected or proven infection caused by any pathogen or clinical sign associated with a high probability of infection, including abnormal temperature or leukocyte count, cough, white blood cells in a normally sterile body fluid, perforated viscus, chest radiograph consistent with pneumonia, petechial or purpuric rash, and purpura fulminans. Neonatal sepsis was defined as the presence of two or more systemic inflammatory response syndrome (SIRS) criteria owing to suspected or proven infection, one being an abnormal temperature or leukocyte count. The criteria for SIRS are as follows: (1) body temperature of more than $38.5^{\circ} \mathrm{C}$ or less than $36^{\circ} \mathrm{C}$; (2) mean heart rate $>2 \mathrm{SD}$ above normal for age in the absence of external stimuli, or unexplained persistent elevation for children $<1$ year old, or mean heart rate $<10$ th percentile for age, or unexplained persistent depression over a $0.5 \mathrm{hr}$ time period; (3) mean respiratory rate of more than $2 \mathrm{SD}$ above normal for age or in the presence of mechanical ventilation; and (4) abnormal leukocyte count or $>10 \%$ immature neutrophils. The detail information can be referred to the published International Pediatric Sepsis Consensus [4].

2.3. Laboratory Measurements. Blood samples were collected on admission to the hospital. WBC count and neutrophil and lymphocyte counts were measured by an automated blood cell counter (Sysmex Corporation, Kobe, Japan). The NLR was calculated by dividing the absolute neutrophil count by the lymphocyte count. High-sensitivity C-reactive protein (hsCRP) was measured using a latex-enhanced immunoturbidimetric assay (Ultrasensitive CRP kit, Upper Bio-Tech,
Shanghai, China) on an UPPER analyzer (Upper Bio-Tech, Shanghai, China). hsCRP levels below $0.8 \mathrm{mg} / \mathrm{L}$ (measurement limits) were considered as $0.7 \mathrm{mg} / \mathrm{L}$. Procalcitonin (PCT) levels were measured using an electrochemiluminescence assay (Elecsys ${ }^{\circledR}$ BRAHMS PCT kit, Roche Diagnostic, Rotkreuz, Switzerland) on a Cobas ${ }^{\circledR} 8000$ modular analyzer (Roche Diagnostic, Rotkreuz, Switzerland). PCT levels above $100 \mathrm{ng} / \mathrm{mL}$ or below $0.02 \mathrm{ng} / \mathrm{mL}$ (measurement limits) were considered as $101 \mathrm{ng} / \mathrm{mL}$ and $0.01 \mathrm{ng} / \mathrm{mL}$, respectively. The levels of serum total bilirubin (TBIL), aspartate aminotransferase (AST), alanine aminotransferase (ALT), total protein (TP), albumin (ALB), alanine aminotransferase (ALT), aspartate aminotransferase (AST), urea nitrogen (UREA), creatinine (CREA), and uric acid (UA) were measured using an automatic biochemistry analyzer (AU5800 Clinical Chemistry Analyzers, Beckman Coulter, California) and a conventional clinical analytical method.

2.4. Statistical Analysis. Statistical analysis was performed using SPSS 21.0 (SPSS Inc., Chicago, Illinois). The normality of variable data was tested prior to further statistical analysis. Normally distributed variables were expressed as the mean \pm standard deviation (SD) and analyzed by independent $t$ -tests or one-way ANOVA, as appropriate. Nonnormally distributed variables were presented as medians (interquartile range) and analyzed using the Mann-Whitney $U$ test. Categorical variables were expressed as number and percentages ( $n, \%)$ and assessed by chi-squared or Fisher exact tests. Analysis of correlations between two continuous variables was performed with a Pearson correlation test. Multivariate logistic regression analysis was performed to identify the independent risk factors for the presence of neonatal sepsis. The risk factors were prespecified, based on univariate $P$ values below 0.05 and previously published literature. Receiver operating characteristic (ROC) curves were performed to evaluate the predictive value of NLR for the presence of neonatal sepsis. Youden's index was calculated $($ sensitivity + specificity -1$)$ to determine the optimal cutoff point. A two-sided $P$ value below 0.05 was considered statistically significant.

\section{Results}

3.1. Baseline Clinical Characteristics of the Study Population. A total of 1480 neonates were enrolled in the study (average age: $8.0(5.0,14.7)$ days; $60.0 \%$ were males $)$ and were divided into 3 groups based on whether they were diagnosed with infection or sepsis. 737 of the neonates were diagnosed with sepsis, and 555 neonates were suspected for having infections. 188 neonates with hyperbilirubinemia were served as controls. Baseline clinical and laboratory data for the three groups are summarized in Table 1.

Neonates with sepsis were older than the neonates with no infection and those with infection $(10.0(5.0,17.0)$ vs. $7.0(5.0,12.0)$ and $7.0(4.0,12.0)$ days, $P<0.001)$. Clinical data showed that neonates with sepsis had a higher body temperature, respiratory rate, and heart rate than controls and neonates with infection $(P<0.001)$. Serum biochemical analysis showed that the levels of PCT, ALT, UREA, and 
TABLE 1: Baseline characteristics of control, infection, and sepsis group.

\begin{tabular}{|c|c|c|c|c|}
\hline Variables & Controls $(n=188)$ & Infection $(n=555)$ & Sepsis $(n=737)$ & $P$ \\
\hline Age (days) & $7.0(5.0,12.0)$ & $7.0(4.0,12.0)$ & $10.0(5.0,17.0)^{\mathrm{bc}}$ & $<0.001$ \\
\hline Male, $n(\%)$ & $115(61.2 \%)$ & $318(57.3 \%)$ & $453(61.5 \%)$ & 0.295 \\
\hline Weight $(\mathrm{kg})$ & $3.3 \pm 0.5$ & $3.3 \pm 0.5$ & $3.2 \pm 0.6^{\mathrm{C}}$ & 0.014 \\
\hline Temperature $\left({ }^{\circ} \mathrm{C}\right)$ & $36.9 \pm 0.3$ & $36.9 \pm 0.4$ & $37.4 \pm 0.8^{\mathrm{bc}}$ & $<0.001$ \\
\hline Respiratory (rate/minute) & $45.4 \pm 6.3$ & $46.3 \pm 7.6$ & $49.8 \pm 10.9^{\mathrm{bc}}$ & $<0.001$ \\
\hline Heart rate $(\mathrm{bpm})$ & $141.8 \pm 12.6$ & $142.4 \pm 15.7$ & $149.7 \pm 19.6^{\mathrm{bc}}$ & $<0.001$ \\
\hline SBP (mm Hg) & $76.9 \pm 7.2$ & $76.4 \pm 6.9$ & $76.1 \pm 8.3$ & 0.439 \\
\hline DBP (mm Hg) & $47.1 \pm 7.5$ & $46.7 \pm 7.1$ & $45.9 \pm 8.0$ & 0.063 \\
\hline PCT (ng/mL) & $0.11(0.09,0.18)$ & $0.14(0.10,0.25)^{\mathrm{a}}$ & $0.31(0.14,1.52)^{\mathrm{bc}}$ & $<0.001$ \\
\hline hsCRP (mg/L) & $0.7(0.7,0.7)$ & $0.7(0.7,0.7)$ & $0.7(0.7,13.48)^{b c}$ & $<0.001$ \\
\hline \multicolumn{5}{|l|}{ Biochemical parameters } \\
\hline TBIL $(\mu \mathrm{mol} / \mathrm{L})$ & $306.4(262.3,357.7)$ & $298.2(230.9,355.8)^{\mathrm{a}}$ & $132.0(45.4,216.1)^{\mathrm{bc}}$ & $<0.001$ \\
\hline $\operatorname{AST}(\mathrm{U} / \mathrm{L})$ & $34.4(28.5,46.8)$ & $37.9(30.2,51.0)^{\mathrm{a}}$ & $38.5(27.9,55.2)$ & 0.078 \\
\hline $\operatorname{ALT}(\mathrm{U} / \mathrm{L})$ & $24.3(17.6,30.3)$ & $25.5(20.0,33.2)$ & $28.5(21.8,37.8)^{\mathrm{bc}}$ & $<0.001$ \\
\hline $\mathrm{TP}(\mathrm{g} / \mathrm{L})$ & $57.5 \pm 5.8$ & $57.3 \pm 6.7$ & $54.1 \pm 7.4^{\mathrm{bc}}$ & $<0.001$ \\
\hline $\operatorname{ALB}(g / L)$ & $34.4 \pm 3.7$ & $33.8 \pm 4.0$ & $30.6 \pm 4.9^{\mathrm{bc}}$ & $<0.001$ \\
\hline UREA (mmol/L) & $2.2(1.3,2.9)$ & $2.3(1.5,3.2)$ & $3.1(1.9,4.4)^{\mathrm{bc}}$ & $<0.001$ \\
\hline CREA $(\mu \mathrm{mol} / \mathrm{L})$ & $49.6(41.0,57.9)$ & $51.0(42.0,59.3)$ & $47.3(37.4,63.1)^{\mathrm{c}}$ & 0.057 \\
\hline $\mathrm{UA}(\mu \mathrm{mol} / \mathrm{L})$ & $135.5(104.9,186.5)$ & $137.9(103.1,182.5)$ & $147.8(109.9,205.0)^{\mathrm{bc}}$ & 0.003 \\
\hline \multicolumn{5}{|l|}{ Hematologic parameters } \\
\hline WBC $\left(10^{9}\right.$ cells $\left./ \mathrm{L}\right)$ & $9.45(7.92,11.67)$ & $10.03(8.10,12.39)$ & $9.99(7.27,14.13)$ & 0.253 \\
\hline Neutrophil count $\left(10^{9}\right.$ cells/L) & $3.72(2.82,5.49)$ & $4.29(3.16,6.07)^{\mathrm{a}}$ & $5.07(3.07,8.58)^{\mathrm{bc}}$ & $<0.001$ \\
\hline Lymphocyte count $\left(10^{9}\right.$ cells $\left./ \mathrm{L}\right)$ & $4.10(3.15,4.86)$ & $3.94(2.95,5.18)$ & $3.34(2.11,4.78)^{\mathrm{bc}}$ & $<0.001$ \\
\hline NLR & $0.92(0.63,1.45)$ & $1.07(0.75,1.84)^{\mathrm{a}}$ & $1.65(0.85,3.07)^{\mathrm{bc}}$ & $<0.001$ \\
\hline
\end{tabular}

All values are presented as the mean \pm SD or $n$ (\%) or as the median (interquartile range). SBP, systolic blood pressure; DBP, diastolic blood pressure; PCT, procalcitonin; TBIL, total bilirubin; AST, aspartate aminotransferase; ALT, alanine aminotransferase; TP, total protein; ALB, albumin; UREA, urea nitrogen; CREA, creatinine; UA, uric acid; WBC, white blood cell; hsCRP, high-sensitivity C-reactive protein; NLR, neutrophil-to-lymphocyte ratio. ${ }^{\mathrm{a}} P<0.05$ for infection vs. control. ${ }^{b} P<0.05$ for sepsis vs. control. ${ }^{\mathrm{c}} P<0.05$ for sepsis vs. infection.

UA were significantly higher in neonates with sepsis $(P<$ 0.05), while serum TBIL, TP, and ALB levels were lower $(P<0.001)$. No differences were found in the levels of AST and CREA among the three groups. In addition, while inflammatory biomarkers such as hsCRP, neutrophil count, lymphocyte count, and NLR were significantly different between the three groups $(P<0.001)$, only PCT, neutrophil count, and NLR showed a gradual increase (Figure 1).

3.2. Association of NLR with the Presence of Neonatal Sepsis. To investigate the association of the NLR levels with the presence of neonatal sepsis, we classified the study participants into three groups according to NLR tertiles. As shown in Table 2 , neonates in tertile 3 were younger $(P<0.001)$ and had a higher body temperature and respiratory rate $(P=0.001)$. In addition, the levels of PCT, hsCRP, UREA, CREA, and UA were significantly higher in tertile 3 $(P<0.001)$, while TBIL, TP, and ALB levels were lower in tertile 3. Further analysis showed that the prevalence of neonatal sepsis increased significantly from $41.6 \%$ in tertile 1 to $66.2 \%$ in tertile 3 group $(P<0.001)$, and neonates with infection were more likely to be in tertile 1 and tertile $2(P<0.001)$.
3.3. Correlation between NLR and Clinical Parameters. To further explore the relationship between the NLR and clinical parameters, we performed Spearman correlation analysis. As shown in Table 3 , NLR was negatively correlated with age $(r=-0.378, P<0.001)$, TBIL $(r=-0.111, P<0.001)$, TP $(r=-0.187, P<0.001)$, and ALB $(r=-0.249, P<0.001)$. Meanwhile, the NLR positively correlated with PCT $(r=0.531, P<0.001)$, hsCRP $(r=0.255, P<0.001)$, UREA $(r=0.251, P<0.001)$, CREA $(r=0.294, P<0.001)$, and UA $(r=0.232, P<0.001)$. In addition, there was no significant correlation between NLR and heart rate, AST, or ALT (all $P>0.05)$.

3.4. Independence of NLR Levels in Predicting Neonatal Sepsis. Univariate and multivariable binary logistic regression analysis was performed to assess the value of NLR in predicting the presence of neonatal sepsis. Variables in univariate analysis with $P<0.05$ were included in a model for multivariate analysis, including age, heart rate, respiratory rate, weight, TP, ALB, CREA, TBIL, PCT, and hsCRP. Multivariate analysis showed that NLR was an independent predictor of the presence of neonatal sepsis (odds ratio $(O R)=1.445,95 \%$ 


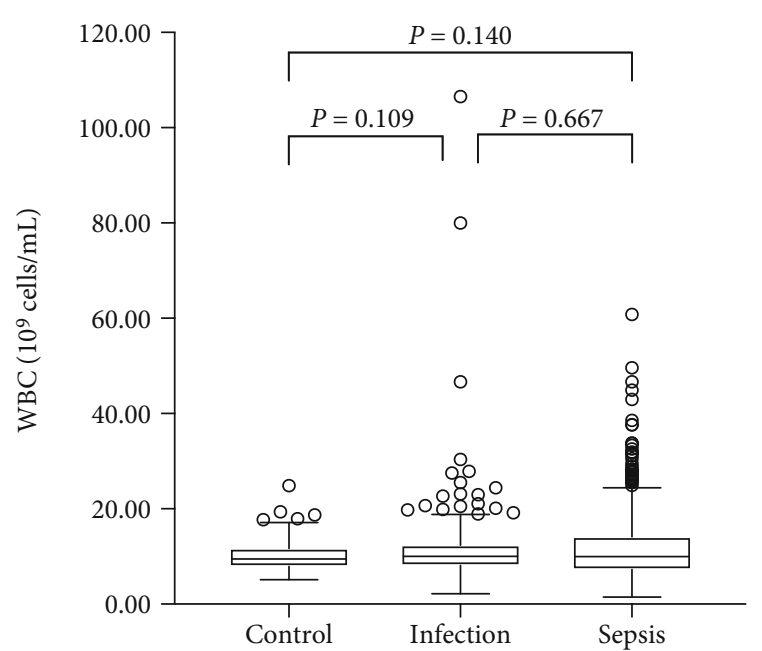

(a)

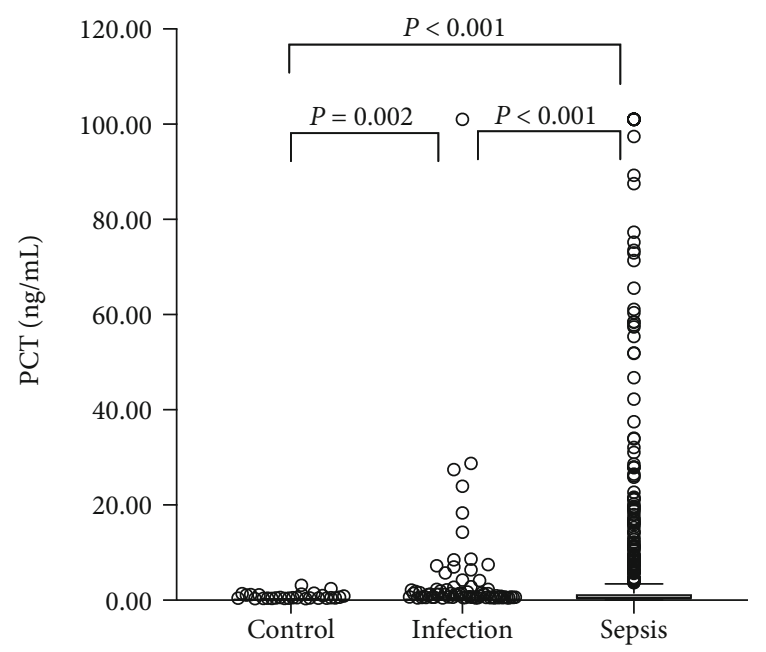

(c)

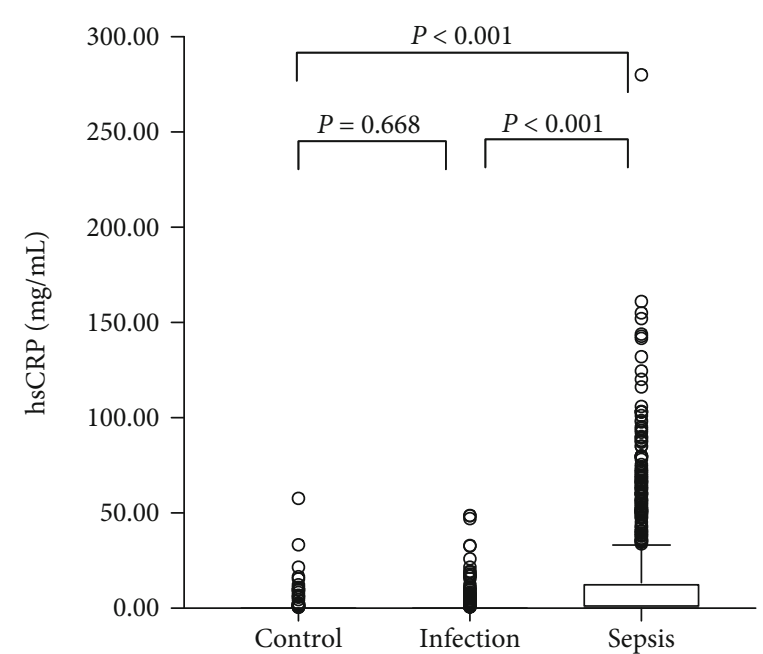

(b)

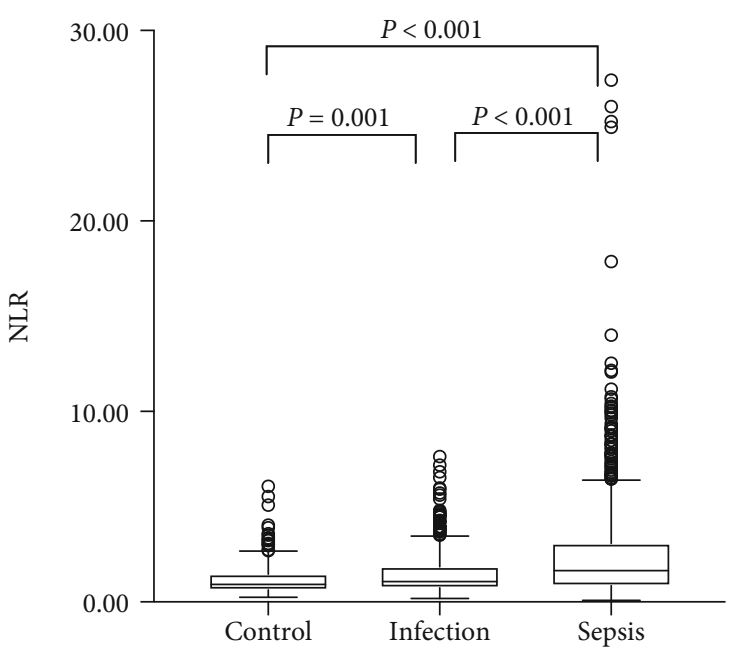

(d)

FIGURE 1: The levels of NLR and other inflammatory markers in control, infection, and sepsis group. (a) WBC had no significant difference among 3 groups. (b) hsCRP significantly increased in neonates with sepsis. (c, d) PCT and NLR showed a gradual increase among 3 groups.

CI 1.301-1.604, $P<0.001$ ) (Table 4). Further analysis showed that NLR was also independent of PCT and hsCRP $(\mathrm{OR}=1.331,95 \%$ CI $1.190-1.604, P<0.001)$. In addition, our multivariate logistic regression models also confirmed that NLR tertiles were also independently associated with an increased prevalence of neonatal sepsis.

3.5. Receiver Operating Characteristic Curve Analysis. Receiver operating characteristic (ROC) curve analysis was performed to evaluate the utility of NLR in predicting the presence of neonatal sepsis. Area under the ROC curve (AUC) indicated a well discriminatory power of NLR (AUC $=0.63,95 \% \mathrm{CI}$ $0.60-0.66, P<0.001)$. Optimal cut-off value of NLR for predicting the presence of neonatal sepsis was 1.62 , with a sensitivity of $51 \%$ and specificity of $75 \%$ (Figure 2 ). According to the cut-off value, we divided the subjects into the two groups (high NLR group $\geq 1.62$ and low NLR group $<1.62$ ). As shown in Figure 3, the high NLR group tended to have higher prevalence of neonatal sepsis and low percentage of no stenosis compared to the low NLR group.

\section{Discussion}

Neonatal sepsis is a serious life-threatening disease in neonates. According to the report by Li et al. [19] in 2015, 2.76 million children worldwide died in the neonatal period, and neonatal sepsis accounted for $15.2 \%$ of these deaths. Early diagnosis of neonatal sepsis is important for early treatment interventions, which in turn can prevent the incidence of serious life-threatening complications and death. Currently, the criteria for diagnosing of neonatal sepsis are mainly based on clinical signs, but these clinical signs are nonspecific [20]. Blood culture is the gold standard but takes up to $48 \mathrm{~h}$ to obtain results. In addition, blood culture is insensitive and can be affected by multiple factors, such as maternal antimicrobial treatment, inadequate volume of blood, and contamination [21]. Identifying rapid, sensitive, and specific new biomarkers is therefore critical.

Neonatal sepsis is a systemic inflammatory response syndrome caused by the invasion of specific or suspected pathogens into the blood and the continuous reproduction of 
TABLe 2: Clinical and demographic characteristics according to NLR tertiles.

\begin{tabular}{|c|c|c|c|c|}
\hline Variables & First tertile $(<0.91)(n=493)$ & First tertile $(0.91-1.88)(n=493)$ & Third tertile $(>1.88)(n=494)$ & $P$ \\
\hline Age (days) & $11.0(7.0,19.0)$ & $8.0(4.0,13.0)^{\mathrm{a}}$ & $5.0(3.0,10.0)^{\mathrm{bc}}$ & $<0.001$ \\
\hline Male, $n(\%)$ & $298(60.4 \%)$ & $290(58.8 \%)$ & $298(60.3 \%)$ & 0.846 \\
\hline PCT (ng/mL) & $0.11(0.09,0.18)$ & $0.16(0.10,0.31)^{\mathrm{a}}$ & $0.50(0.21,2.86)^{\mathrm{bc}}$ & $<0.001$ \\
\hline hsCRP (mg/L) & $0.7(0.7,0.7)$ & $0.7(0.7,0.7)$ & $0.7(0.7,15.53)^{\mathrm{bc}}$ & $<0.001$ \\
\hline \multicolumn{5}{|c|}{ Biochemical parameters } \\
\hline TBIL $(\mu \mathrm{mol} / \mathrm{L})$ & $258.9(93.7,335.3)$ & $260.8(126.2,330.0)$ & $191.2(111.1,278.3)^{\mathrm{bc}}$ & $<0.001$ \\
\hline AST (U/L) & $37.0(29.1,51.7)$ & $36.1(28.3,48.3)$ & $40.3(29.3,57.1)^{\mathrm{c}}$ & 0.009 \\
\hline $\operatorname{ALT}(\mathrm{U} / \mathrm{L})$ & $26.7(21.4,35.7)$ & $25.5(19.3,33.1)^{\mathrm{a}}$ & $27.8(21.0,36.6)^{\mathrm{c}}$ & 0.005 \\
\hline $\mathrm{TP}(\mathrm{g} / \mathrm{L})$ & $57.0 \pm 6.3$ & $56.1 \pm 7.6$ & $54.0 \pm 7.1^{\mathrm{bc}}$ & $<0.001$ \\
\hline $\operatorname{ALB}(g / L)$ & $33.4 \pm 4.4$ & $32.6 \pm 4.6^{\mathrm{a}}$ & $30.7 \pm 4.9^{\mathrm{bc}}$ & $<0.001$ \\
\hline UREA (mmol/L) & $2.3(1.4,3.2)$ & $2.4(1.6,3.9)^{\mathrm{a}}$ & $3.1(2.1,4.7)^{\mathrm{bc}}$ & $<0.001$ \\
\hline CREA $(\mu \mathrm{mol} / \mathrm{L})$ & $44.8(37.2,52.5)$ & $48.8(40.0,58.1)^{\mathrm{a}}$ & $57.0(43.1,75.7)^{\mathrm{bc}}$ & $<0.001$ \\
\hline $\mathrm{UA}(\mu \mathrm{mol} / \mathrm{L})$ & $130.3(100.6,159.9)$ & $138.9(104.5,185.4)^{\mathrm{a}}$ & $170.1(118.9,253.5)^{\mathrm{bc}}$ & $<0.001$ \\
\hline \multicolumn{5}{|l|}{ Clinical data } \\
\hline Control, $n(\%)$ & $91(18.5 \%)$ & $65(13.2 \%)$ & $32(6.5 \%)$ & $<0.001$ \\
\hline Infection & $197(40.0 \%)$ & $223(45.2 \%)$ & $135(27.3 .0 \%)^{\mathrm{bc}}$ & $<0.001$ \\
\hline Sepsis & $205(41.6 \%)$ & $205(41.6 \%)$ & $327(66.2 \%)^{b c}$ & $<0.001$ \\
\hline
\end{tabular}

Abbreviations as in Table $1 .{ }^{b} P<0.05$ for sepsis vs. control. ${ }^{c} P<0.05$ for sepsis vs. infection.

TABLE 3: Correlations between NLR and clinical parameters.

\begin{tabular}{lcc}
\hline Variables & $r$ & $P$ \\
\hline Age $($ day $)$ & -0.378 & $<0.001$ \\
PCT $(\mathrm{ng} / \mathrm{mL})$ & 0.531 & $<0.001$ \\
$\operatorname{hsCRP}(\mathrm{mg} / \mathrm{L})$ & 0.255 & $<0.001$ \\
TBIL $(\mu \mathrm{mol} / \mathrm{L})$ & -0.111 & $<0.001$ \\
AST $(\mathrm{U} / \mathrm{L})$ & 0.050 & 0.053 \\
ALT $(\mathrm{U} / \mathrm{L})$ & 0.023 & 0.385 \\
TP $(\mathrm{g} / \mathrm{L})$ & -0.187 & $<0.001$ \\
ALB $(\mathrm{g} / \mathrm{L})$ & -0.249 & $<0.001$ \\
UREA $(\mathrm{mmol} / \mathrm{L})$ & 0.251 & $<0.001$ \\
CREA $(\mu \mathrm{mol} / \mathrm{L})$ & 0.294 & $<0.001$ \\
UA $(\mu \mathrm{mol} / \mathrm{L})$ & 0.232 & $<0.001$ \\
\hline
\end{tabular}

Abbreviations as in Table 1.

toxins. It is accompanied by pathological inflammation and organ system dysfunction [1]. Neutrophils are an essential arm of the innate immune response during sepsis and release inflammatory cytokines, chemokines, and regulatory cytokines. Neutrophils can also engulf invading pathogens and kill them via a range of antimicrobial peptides, proteases, and oxidants [22]. In recent years, the discovery of neutrophil extracellular traps (NETs) has uncovered a new weapon in the immune system defenses against pathogen infection [23-25]. However, excessive expression of inflammatory cytokines and NET formation contributes to excessive inflammation and tissue damage [26-28]. Lymphocytes are also involved in the immune response against infections by bacteria and viruses. During pathogen infection, antigenpresenting cells recognized microbial antigens and presented it to $\mathrm{T}$ cells. Subsequently, $\mathrm{CD} 4^{+} \mathrm{T}$ cells secrete cytokines helping phagocytotic cells to kill intracellular bacteria [29] However, during sepsis, the number of lymphocytes declines significantly due to apoptosis. This decline is considered an important contributing factor to the immunosuppressive state that makes patients vulnerable to new infections [30,31].

Total and differential leukocyte counts represented cheap, widely available indicators of the inflammatory response. NLR reflects changes in neutrophil and lymphocyte counts. Many studies have demonstrated that NLR represents a reliable inflammatory marker and prognostic index in a variety of medical conditions, including ischemic stroke $[32,33]$, cerebral hemorrhage $[34,35]$, major adverse cardiac events [36-39], and solid tumors [40, 41]. Recently, the NLR has attracted substantial attention as a new risk factor with potential for use in the diagnosis of sepsis. Sepsis could give rise to elevated neutrophil counts and decreased lymphocyte counts resulting from the infection of pathogenic microorganisms, indicating that sepsis patients might have a higher NLR level [10, 42-44]. Numerous epidemiological investigations and meta-analysis studies have demonstrated that NLR may be a helpful predictor of sepsis, and patients with higher NLR may have a higher risk of unfavorable prognosis [12, 45]. However, most studies on the relationship between NLR and sepsis have been conducted in adults. There are only a few published studies (based on small sample sizes, $n$ $<150$ ) showing that NLR is positively correlated with neonatal sepsis [15-18].

In the current study, we enrolled a relatively large patient population (1480 neonates) and found that patients in the neonatal sepsis group had the highest level of NLR. To investigate the association of NLR levels with the presence of neonatal sepsis, subjects were divided into three groups according to NLR tertiles. Further analysis showed that the prevalence of neonatal sepsis showed a progressive increase 
TABLE 4: Multivariate logistic regression analysis for prediction of neonatal sepsis.

\begin{tabular}{lccc}
\hline Model & Variables & OR $(95 \%$ CI $)$ & $P$ \\
\hline \multirow{4}{*}{ Model 1 } & NLR & $1.450(1.340-1.569)$ & $<0.001$ \\
& NLR tertiles & & \\
& Tertile 1 & 1 & \\
& Tertile 2 & $1.000(0.776-1.288)$ & 1.000 \\
& Tertile 3 & $2.751(2.124-3.562)$ & $<0.001$ \\
\hline \multirow{4}{*}{ Model 2 } & NLR & $1.445(1.301-1.604)$ & $<0.001$ \\
& NLR tertiles & & \\
& Tertile 1 & 1 & 0.511 \\
& Tertile 2 & $1.116(0.804-1.550)$ & $<0.001$ \\
\hline Tertile 3 & $2.796(1.941-4.029)$ & $<0.001$ \\
& NLR & $1.331(1.190-1.489)$ & \\
Model 3 & NLR tertiles & & 0.938 \\
& Tertile 1 & 1 & $<0.001$ \\
\hline & Tertile 2 & $0.987(0.707-1.378)$ & \\
& Tertile 3 & $2.039(1.395-2.980)$ &
\end{tabular}

Abbreviations as in Table 1. Model 1: unadjusted. Model 2: adjusted for age, heart rate, respiratory rate, weight, TP, ALB, CREA, and TBIL. Model 3: adjusted for age, heart rate, respiratory rate, weight, TP, ALB, CREA, TBIL, PCT, and hsCRP.

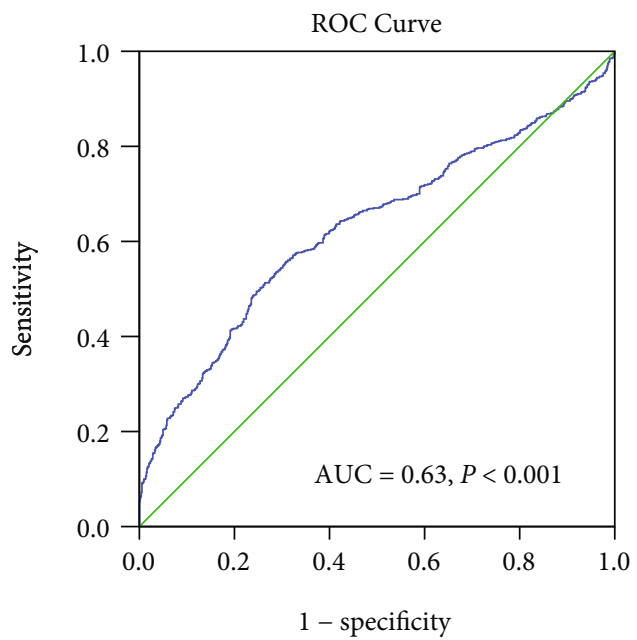

FIGURE 2: Receiver operating characteristic (ROC) curves of NLR to predict the presence of neonatal sepsis.

from NLR tertile 1 to tertile 3. Multivariate analysis showed that NLR was an independent predictor of the presence of neonatal sepsis. Moreover, the ROC curve inferred an NLR cut-off value of 1.62 in predicting the presence of neonatal sepsis. Like NLR, PCT and hsCRP are also inflammatory markers, and many studies have demonstrated that elevated PCT and hsCRP were useful biomarkers for the diagnosis of neonatal sepsis, and elevated PCT and hsCRP were associated with the presence of neonatal sepsis [46-48]. In agreement with previous reports, our study also demonstrated that PCT and hsCRP are independently associated with the presence of neonatal sepsis (data not shown). Nonetheless,

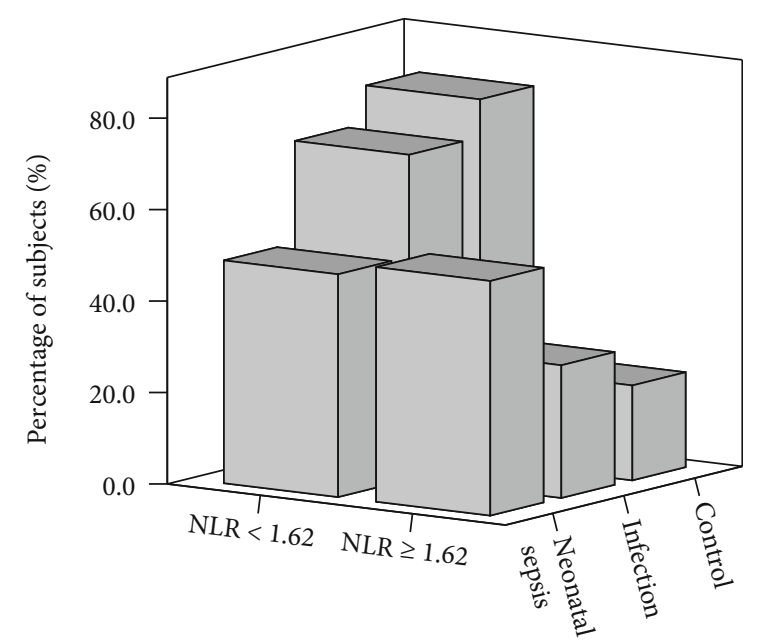

FIgURE 3: The distribution of subjects in high $(\geq 1.62)$ or low $(<$ 1.62) NLR groups.

NLR was still an independent risk factor for neonatal sepsis when PCT and CRP were added to the multivariate regression model.

There are several limitations in our study. First, this is a cross-sectional and single-center study, which cannot predict future events and may have some inherent biases. Second, the diagnosis of neonatal sepsis was based on clinical features and was not confirmed by positive blood culture. Third, NLR was only measured at one time point. Serial measurements of NLR and the change in neonatal sepsis would provide more information on their relationship and would be useful for further exploring the dynamic correlation between them.

\section{Conclusion}

The current study demonstrated that there existed a relationship between NLR and the presence of neonatal sepsis. NLR level was higher in patients with neonatal sepsis and showed a gradual increase among three groups. Meanwhile, multivariate analysis showed that NLR was independently associated with the presence of neonatal sepsis. The findings highlight the potential value of NLR in predicting the risk of neonatal sepsis.

\section{Data Availability}

The data used to support the findings of this study are available from the corresponding author upon request.

\section{Conflicts of Interest}

The authors declare that they have no conflicts of interest.

\section{Authors' Contributions}

Tiewei Li and Geng Dong have contributed equally to this work and should be considered co-first authors. 


\section{Acknowledgments}

This work was supported by the Key Research, Development, and Promotion Projects of Henan Province (202102310132) and the Medical Science and Technology Project of Henan Province (2018020698).

\section{References}

[1] A. L. Shane, P. J. Sánchez, and B. J. Stoll, "Neonatal sepsis," The Lancet, vol. 390, no. 10104, pp. 1770-1780, 2017.

[2] S. A. Qazi and B. J. Stoll, "Neonatal sepsis: a major global public health challenge," The Pediatric Infectious Disease Journal, vol. 28, Supplement, pp. S1-S2, 2009.

[3] C. Fleischmann-Struzek, D. M. Goldfarb, P. Schlattmann, L. J. Schlapbach, K. Reinhart, and N. Kissoon, "The global burden of paediatric and neonatal sepsis: a systematic review," The Lancet Respiratory Medicine, vol. 6, no. 3, pp. 223-230, 2018.

[4] B. Goldstein, B. Giroir, and A. Randolph, "International pediatric sepsis consensus conference: definitions for sepsis and organ dysfunction in pediatrics," Pediatric Critical Care Medicine, vol. 6, no. 1, pp. 2-8, 2005.

[5] C. S. Scheer, C. Fuchs, M. Gründling et al., "Impact of antibiotic administration on blood culture positivity at the beginning of sepsis: a prospective clinical cohort study," Clinical Microbiology and Infection, vol. 25, no. 3, pp. 326-331, 2019.

[6] B. Lamy, S. Dargère, M. C. Arendrup, J. J. Parienti, and P. Tattevin, "How to optimize the use of blood cultures for the diagnosis of bloodstream infections? A state-of-the art," Frontiers in Microbiology, vol. 7, p. 697, 2016.

[7] D. Sharma, N. Farahbakhsh, S. Shastri, and P. Sharma, "Biomarkers for diagnosis of neonatal sepsis: a literature review," The Journal of Maternal-Fetal \& Neonatal Medicine, vol. 31, no. 12, pp. 1646-1659, 2018.

[8] D. Djordjevic, G. Rondovic, M. Surbatovic et al., "Neutrophilto-lymphocyte ratio, monocyte-to-lymphocyte ratio, plateletto- lymphocyte ratio, and mean platelet volume-to-platelet count ratio as biomarkers in critically ill and injured patients: which ratio to choose to predict outcome and nature of bacteremia?," Mediators of Inflammation, vol. 2018, Article ID 3758068, 15 pages, 2018.

[9] E. C. Martins, L. D. F. Silveira, K. Viegas et al., "Neutrophillymphocyte ratio in the early diagnosis of sepsis in an intensive care unit: a case-control study," Revista Brasileira de Terapia Intensiva, vol. 31, no. 1, pp. 64-70, 2019.

[10] R. de Pablo, J. Monserrat, A. Prieto, and M. Alvarez-Mon, "Role of circulating lymphocytes in patients with sepsis," BioMed Research International, vol. 2014, Article ID 671087, 11 pages, 2014.

[11] A. Dirican, B. B. Kucukzeybek, A. Alacacioglu et al., "Do the derived neutrophil to lymphocyte ratio and the neutrophil to lymphocyte ratio predict prognosis in breast cancer?," International Journal of Clinical Oncology, vol. 20, no. 1, pp. 70-81, 2015.

[12] Z. Huang, Z. Fu, W. Huang, and K. Huang, "Prognostic value of neutrophil-to-lymphocyte ratio in sepsis: a meta-analysis," The American Journal of Emergency Medicine, vol. 38, no. 3, pp. 641-647, 2020.

[13] S. Y. Hwang, T. G. Shin, I. J. Jo et al., "Neutrophil-to-lymphocyte ratio as a prognostic marker in critically-ill septic patients," The American Journal of Emergency Medicine, vol. 35, no. 2, pp. 234-239, 2017.
[14] X. Liu, Y. Shen, H. Wang, Q. Ge, A. Fei, and S. Pan, "Prognostic significance of neutrophil-to-lymphocyte ratio in patients with sepsis: a prospective observational study," Mediators of Inflammation, vol. 2016, Article ID 8191254, 8 pages, 2016.

[15] S. Alkan Ozdemir, E. Arun Ozer, O. Ilhan, and S. Sutcuoglu, "Can neutrophil to lymphocyte ratio predict late-onset sepsis in preterm infants?," Journal of Clinical Laboratory Analysis, vol. 32, no. 4, article e22338, 2018.

[16] A. Omran, A. Maaroof, M. H. S. Mohammad, and A. Abdelwahab, "Proteina $C$ reativa salivar, volume medio de plaquetas e proporçao de neutrofilos/linfocitos como marcadores de diagnostico de sepse neonatal," Jornal de Pediatria, vol. 94, no. 1, pp. 82-87, 2018.

[17] E. Can, S. Hamilcikan, and C. Can, "The value of neutrophil to lymphocyte ratio and platelet to lymphocyte ratio for detecting early-onset neonatal sepsis," Journal of Pediatric Hematology/Oncology, vol. 40, no. 4, pp. e229-e232, 2018.

[18] J. H. Lee, "Eosinophil count and neutrophil-to-lymphocyte count ratio as biomarkers for predicting early-onset neonatal sepsis," Korean Journal of Pediatrics, vol. 62, no. 12, pp. 438439, 2019.

[19] L. Liu, S. Oza, D. Hogan et al., "Global, regional, and national causes of child mortality in 2000-13, with projections to inform post-2015 priorities: an updated systematic analysis," The Lancet, vol. 385, no. 9966, pp. 430-440, 2015.

[20] S. Mukhopadhyay and K. M. Puopolo, "Risk assessment in neonatal early onset sepsis," Seminars in Perinatology, vol. 36, no. 6, pp. 408-415, 2012.

[21] P. Y. Iroh Tam and C. M. Bendel, "Diagnostics for neonatal sepsis: current approaches and future directions," Pediatric Research, vol. 82, no. 4, pp. 574-583, 2017.

[22] M. A. Kovach and T. J. Standiford, "The function of neutrophils in sepsis," Current Opinion in Infectious Diseases, vol. 25, no. 3, pp. 321-327, 2012.

[23] R. H. L. Li and F. Tablin, "A comparative review of neutrophil extracellular traps in sepsis," Frontiers in Veterinary Science, vol. 5, p. 291, 2018.

[24] V. Brinkmann, U. Reichard, C. Goosmann et al., "Neutrophil extracellular traps kill bacteria," Science, vol. 303, no. 5663, pp. 1532-1535, 2004.

[25] B. McDonald, R. Urrutia, B. G. Yipp, C. N. Jenne, and P. Kubes, "Intravascular neutrophil extracellular traps capture bacteria from the bloodstream during sepsis," Cell Host of Microbe, vol. 12, no. 3, pp. 324-333, 2012.

[26] P. G. Czaikoski, J. M. S. C. Mota, D. C. Nascimento et al., "Neutrophil extracellular traps induce organ damage during experimental and clinical sepsis," PLoS One, vol. 11, no. 2, article e0148142, 2016.

[27] T. van der Poll, F. L. van de Veerdonk, B. P. Scicluna, and M. G. Netea, "The immunopathology of sepsis and potential therapeutic targets," Nature Reviews Immunology, vol. 17, no. 7, pp. 407-420, 2017.

[28] T. Li, Z. Zhang, X. Li et al., "Neutrophil extracellular traps: signaling properties and disease relevance," Mediators of Inflammation, vol. 2020, Article ID 9254087, 14 pages, 2020.

[29] D. J. Stearns-Kurosawa, M. F. Osuchowski, C. Valentine, S. Kurosawa, and D. G. Remick, "The pathogenesis of sepsis," Annual Review of Pathology, vol. 6, no. 1, pp. 19-48, 2011.

[30] Y. Le Tulzo, C. Pangault, A. Gacouin et al., "Early circulating lymphocyte apoptosis in human septic shock is associated with poor outcome," Shock, vol. 18, no. 6, pp. 487-494, 2002. 
[31] R. S. Hotchkiss and I. E. Karl, "The pathophysiology and treatment of sepsis," The New England Journal of Medicine, vol. 348, no. 2, pp. 138-150, 2003.

[32] A. Celikbilek, S. Ismailogullari, and G. Zararsiz, "Neutrophil to lymphocyte ratio predicts poor prognosis in ischemic cerebrovascular disease," Journal of Clinical Laboratory Analysis, vol. 28, no. 1, pp. 27-31, 2014.

[33] Y. L. Liu, J. K. Lu, H. P. Yin et al., "High neutrophil-tolymphocyte ratio predicts hemorrhagic transformation in acute ischemic stroke patients treated with intravenous thrombolysis," International Journal of Hypertension, vol. 2020, Article ID 5980261, 6 pages, 2020.

[34] S. Lattanzi, F. Brigo, E. Trinka, C. Cagnetti, M. di Napoli, and M. Silvestrini, "Neutrophil-to-lymphocyte ratio in acute cerebral hemorrhage: a system review," Translational Stroke Research, vol. 10, no. 2, pp. 137-145, 2019.

[35] S. Lattanzi, C. Cagnetti, C. Rinaldi, S. Angelocola, L. Provinciali, and M. Silvestrini, "Neutrophil-to-lymphocyte ratio improves outcome prediction of acute intracerebral hemorrhage," Journal of the Neurological Sciences, vol. 387, pp. 98102, 2018.

[36] J. S. Park, K. W. Seo, B. J. Choi et al., "Importance of prognostic value of neutrophil to lymphocyte ratio in patients with stelevation myocardial infarction," Medicine, vol. 97, no. 48, article e13471, 2018.

[37] U. U. Tamhane, S. Aneja, D. Montgomery, E. K. Rogers, K. A. Eagle, and H. S. Gurm, "Association between admission neutrophil to lymphocyte ratio and outcomes in patients with acute coronary syndrome," The American Journal of Cardiology, vol. 102, no. 6, pp. 653-657, 2008.

[38] M. E. Afari and T. Bhat, "Neutrophil to lymphocyte ratio (nlr) and cardiovascular diseases: an update," Expert Review of Cardiovascular Therapy, vol. 14, no. 5, pp. 573-577, 2016.

[39] X. Wang, X. Fan, S. Ji, A. Ma, and T. Wang, "Prognostic value of neutrophil to lymphocyte ratio in heart failure patients," Clinica Chimica Acta, vol. 485, pp. 44-49, 2018.

[40] A. J. Templeton, M. G. McNamara, B. Šeruga et al., "Prognostic role of neutrophil-to-lymphocyte ratio in solid tumors: a systematic review and meta-analysis," Journal of the National Cancer Institute, vol. 106, no. 6, 2014.

[41] J. L. Ethier, D. Desautels, A. Templeton, P. S. Shah, and E. Amir, "Prognostic role of neutrophil-to-lymphocyte ratio in breast cancer: a systematic review and meta-analysis," Breast Cancer Research, vol. 19, no. 1, p. 2, 2017.

[42] B. Tomar, H. J. Anders, J. Desai, and S. R. Mulay, "Neutrophils and neutrophil extracellular traps drive necroinflammation in COVID-19," Cells, vol. 9, no. 6, p. 1383, 2020.

[43] X. F. Shen, K. Cao, J. P. Jiang, W. X. Guan, and J. F. Du, "Neutrophil dysregulation during sepsis: an overview and update," Journal of Cellular and Molecular Medicine, vol. 21, no. 9, pp. 1687-1697, 2017.

[44] A. M. Drewry, N. Samra, L. P. Skrupky, B. M. Fuller, S. M. Compton, and R. S. Hotchkiss, "Persistent lymphopenia after diagnosis of sepsis predicts mortality," Shock, vol. 42, no. 5, pp. 383-391, 2014.

[45] J. Ni, H. Wang, Y. Li, Y. Shu, and Y. Liu, "Neutrophil to lymphocyte ratio (nlr) as a prognostic marker for in-hospital mortality of patients with sepsis: a secondary analysis based on a single-center, retrospective, cohort study," Medicine, vol. 98, no. 46, article e18029, 2019.
[46] D. W. Jekarl, S. Lee, M. Kim, Y. Kim, S. H. Woo, and W. J. Lee, "Procalcitonin as a prognostic marker for sepsis based on sepsis-3," Journal of Clinical Laboratory Analysis, vol. 33, no. 9, article e22996, 2019.

[47] S. L. Fan, N. S. Miller, J. Lee, and D. G. Remick, "Diagnosing sepsis - the role of laboratory medicine," Clinica Chimica Acta, vol. 460, pp. 203-210, 2016.

[48] S. Eschborn and J. H. Weitkamp, "Procalcitonin versus creactive protein: review of kinetics and performance for diagnosis of neonatal sepsis," Journal of Perinatology, vol. 39, no. 7, pp. 893-903, 2019. 